\section{Institutional \\ Solutions to \\ Enhance Power \\ Sector Resilience}

How will power system operators, personnel, and end users react during a major disruption? Will system operations adapt to changing conditions and recover quickly? A balanced approach to power sector resilience that includes both institutional and technical solutions will help answer these questions. Institutional solutions include:

- Building human capacity

- Improving access to data

- Enhancing operational intelligence

- Engaging stakeholders

- Developing a culture of resilience

- Implementing other relevant institutional operational processes.

Control center operators and power sector personnel will ultimately oversee the response to any major power system event, and their knowledge, skills, and training, in addition to the tools available to them, are critical to effectively managing any event (National Academies of Sciences, Engineering, and Medicine 2017). A recent global sector survey from the World Bank (2016) identified low awareness of threat exposure and risk management standards

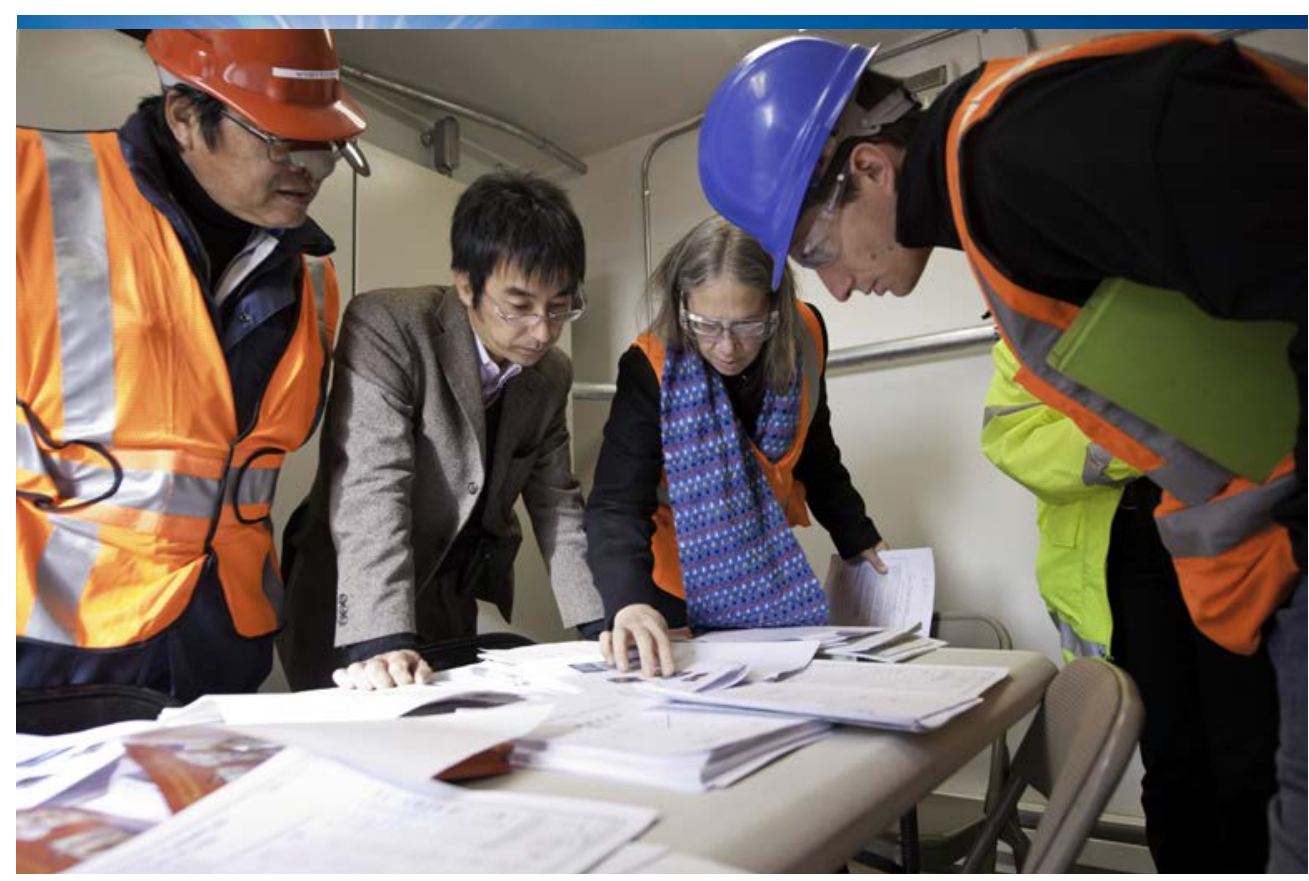

Figure 1. Institutional solutions that enhance operational intelligence and engage stakeholders often have a greater return on investment for resilience than purely technical solutions and should be considered in any holistic resilience planning activity (Wang et al. 2016). Photo by Dennis Schroeder, NREL 19109

and weak institutional capacity as dominant barriers to increased power sector resilience. There is a need to prioritize the design of systems and institutional processes as well as the sharing and linking of relevant data to improve resilience (Foster 2017). These concerns highlight the importance of implementing institutional solutions in addition to technical solutions to support power sector resilience.

While technical solutions (such as mini grid development, storage, critical asset relocation, and others) may be more familiar to power sector personnel, institutional solutions improve the capabilities of power system operators and power sector personnel to anticipate, prepare for, and respond quickly and appropriately to the impacts of power system threatsenhancing power sector resilience. Institutional solutions are also often the most cost effective option and should be considered in any holistic resilience planning activity (Wang et al. 2016). The following sections highlight key institutional solutions to enable power system resilience.

\section{Build Knowledge and Capacity of Personnel}

Power system operators are responsible-to some degree-for addressing outcomes of every major event that impacts the power 


\section{Glossary}

Critical loads: Loads for which power supply must always be maintained without interruption to ensure the functioning of society and the economy, such as hospitals, critical communications, emergency services, water and wastewater treatment, and military installations.

Flexibility (operational): The ability of a power system to respond to changes in electricity demand and supply. High flexibility implies that a system can respond quickly to changes in net load.

Impact: The extent to which a threat affects power sector infrastructure and processes (e.g., a typhoon causes wind damage to transmission lines). This term is often used interchangeably with consequence or outcome.

Power Sector Resilience: The ability to anticipate, prepare for, and adapt to changing conditions and withstand, respond to, and recover rapidly from disruptions to the power sector through adaptable and holistic planning and technical solutions (Hotchkiss et al. 2018).

Threat: Anything that can damage, destroy, or disrupt the power sector. Threats can be natural, human caused, or technological. Threats are not typically within the control of power system planners and operators. They may consist of wildfires, hurricanes, storm surges, cyberattacks, and others.

\section{Vulnerability: Weaknesses} within infrastructure, processes, or systems, or the degree of susceptibility to various threats. Different measures can be taken to reduce vulnerability or improve adaptive capacity to threats to the power sector.

\section{Tonga Cyclone Highlights Need for Response Plans and Training for Major Events}

In 2014, Tonga, an island nation in the South Pacific with around 7,000 inhabitants, was hit by tropical cyclone lan, a Category 5 cyclone with wind speeds up to 287 kilometers per hour. This powerful storm destroyed approximately $82 \%$ of all buildings and $95 \%$ of power lines in addition to damaging the only power station. Response efforts following the storm were slowed due to a lack of functioning communication systems to support Tonga Power Limited, the country's sole electricity provider, in assessing the damages and needs as well as coordinating the response. After 2 weeks, power was restored to fewer than 100 households, and after 10 months almost $50 \%$ of the population was still without power (World Bank 2016).

A survey of Tonga Power Limited managers following this event offered suggestions for improving power system resilience for future events. Managers noted that predisaster preparation and system maintenance were critical. In particular, managers identified the need to compile a restoration priority list detailing the critical systems for each section of the power network as well as a broader response plan. This response plan would compile existing knowledge of the system and form a basis for operator and personnel training for future extreme events (World Bank 2016). 
system. To ensure a resilient power system, personnel need to know what to do prior to, during, and following the possible exposure of a vulnerability. This requires education and training to support well-informed decisions based on best practices and high-quality, robust data (National Academies of Sciences, Engineering, and Medicine 2017).

Knowledge and capacity-building of power system operators can build off of existing plans for extreme events, continuity of operations plans, or similar response plans. Beyond outlining critical steps to be taken following an extreme event, response plans can ensure that responsibilities are clearly defined and that personnel can complete their tasks given any of a wide array of contingencies. This requires training, not only for the normal operating conditions of a power system, but also training with an emphasis on what to do during operational power system stages of alert, emergency, extreme conditions, and restoration (National Academies of Sciences, Engineering, and Medicine 2017). Box 1 describes work in Tonga to develop a response plan that informed related training needs.

Response and resilience trainings can also bring together stakeholders across the power sector to simulate events that impact the power system.
For example, power system operators can coordinate and conduct disaster scenario exercises with emergency response managers, utilities, and critical load owners to practice response actions based on established response plans. These trainings also support stakeholder engagement and improved organizational communication.

\section{Improve Access to Weather and Climate Data}

\begin{abstract}
Many risks to the power sector are weather and climate driven. As such, use of predictive weather and climate models - at a range of spatial and temporal scales-is often a strategic
\end{abstract}

\section{France and Norway Utilities Enhance Resilience with Climate and Weather Data}

\section{Short-term Weather Forecasts}

Enedis, formally ERDF, a distribution network of Électricité de France (EDF) uses a weather monitoring and modeling tool called Geriko. The tool helps to evaluate potential shortterm weather-related risks (such as high winds, snow, and icing, among others) to the distribution system. The Geriko tool helped warn Enedis about the windstorm Joachim, which had gusts of up to 150 kilometers per hour that affected Western Europe in December 2011. With advance warning, Enedis was able to put personnel on standby for a potential event and restore service within 24 hours to $95 \%$ of the 700,000 customers who lost power following the storm (WBCSD 2014; Insurance Journal 2011).

\section{Medium- and Long-term Weather and Climate Forecasts}

Statkraft, a hydropower generation company in Norway, has long used historical data to support operations and long-term planning activities. However, in response to recent shifting seasonal patterns in rain and snow in the country, Statkraft now relies on more recent historical data that reflect these changes to ensure they can appropriately forecast future precipitation-using historical data from 1980 to 2010 as opposed to 1960 to 1990 . To support longterm planning, Statkraft combines historical data with results from global climate models to capture potential patterns to 2100 along the lifetime of generation assets.

Statkraft also collaborates with the Norwegian meteorological institute to understand the potential effects of climate change (such as precipitation and temperature) in Nordic countries. The goal is to understand how the results of global and regional meteorological models can be applied at the hydropower basin-level scale. For example, changes in precipitation, snowfall, and temperature significantly influence the operation of high mountain hydropower generation and specifically seasonal runoff and generation patterns. Statkraft also works with the Norwegian Water Authority to develop methodologies that help forecast changes in temperature and precipitation to understand how a changing climate may affect hydropower resource availability. These methodologies and improved climate data have helped Statkraft develop a better understanding of potential reservoir inflow patterns and hydropower generation resources and adjust their simulations, operations planning, and long-term price forecasts (WBCSD 2014).

EDF models hydrological and thermal systems along the Rhone river basin to support long-term planning to 2030, 2050, and 2085 to understand water resources in Lake Geneva, nuclear generation system operations, and regional climate simulations. These models highlight potential mean water temperatures and precipitation and snow melt, which may affect both thermal and hydropower generation in the river basin (WBCSD 2014). 


\section{Florida Utility Prioritizes Operational Intelligence to Ensure Readiness, Restoration, and Recovery}

Florida Power and Light Company (FPL) is one of the largest utilities in the United States with more than 10 million customers across Florida. To enhance power system resilience, FPL has developed a comprehensive storm and disaster response plan that focuses on institutional readiness, restoration, and recovery.

\section{FPL's response plan improves} institutional readiness through annual trainings that include major storm simulations and emergency response practice across the utility's footprint. To ensure rapid restoration, FPL has agreements with out-of-state utilities that can provide additional workers to respond to the impacts of threats. The response plan requires the storage of backup supplies and equipment and strategic staging of this material throughout their service area to support quick restoration and recovery of services. FPL also coordinates with stakeholders such as local emergency officials to identify critical loads that require power during and after major events (such as hospitals, police and fire stations, communication infrastructure, water treatment, and transportation providers).

Additionally, FPL works to prepare and strengthen its infrastructure (technical solutions) throughout the year by inspecting, maintaining (including the clearing of tree branches from routes), repairing, and upgrading power lines and other infrastructure (FPL 2019). operational addition to resilience planning. These models can be used in a variety of ways. Weather-driven demand forecasting may be tied to long-, medium-, and short-term weather forecasts to more accurately predict consumer behavior under expected conditions (Dehghanian et al. 2019). With sufficient warning of threats such as hurricanes and severe weather, operators can limit avoidable outages (such as planned maintenance), deploy additional reserves, and order preemptive disconnections of systems that are at risk to ensure the system is robust and prepared for potential impacts. These capabilities are often less expensive than the hardening of physical infrastructure (National Academies of Sciences, Engineering, and Medicine 2017). Long- and medium-term weather and climate forecasts may be used to prepare the system for predicted major events that will have months-long impacts to electricity consumption and generation (such as El Niño or La Niña patterns). Shorter-term forecasts may be used to

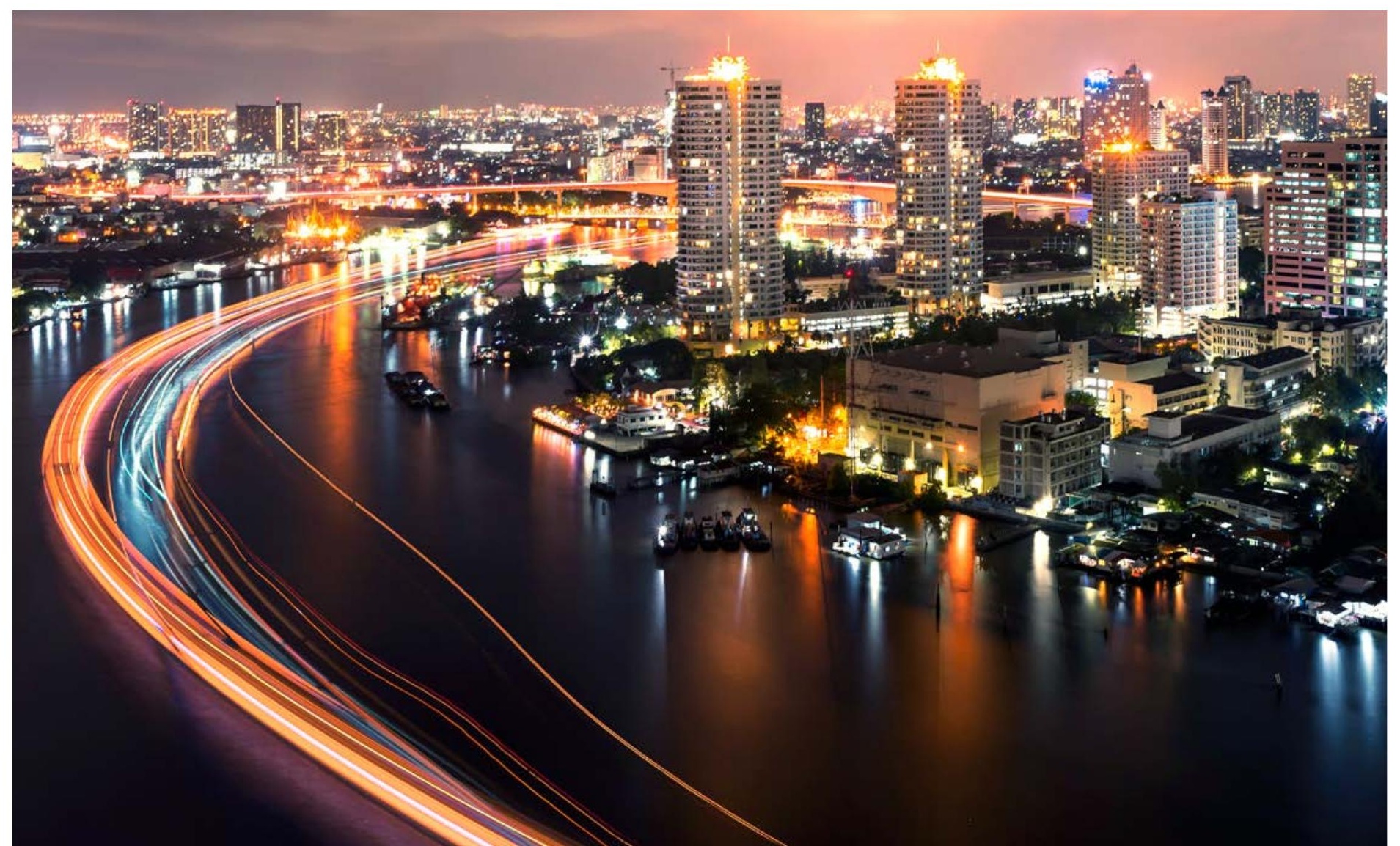


route power around localized and/or acute events that may impact power systems (such as expected flooding or heat waves). These weather and demand forecasts may also trigger demand response protocols that relate to increases in load.

Power system modeling (including longterm capacity expansion, production cost modeling, and generation system performance models, among others) also benefits from the inclusion of short-, medium-, and long-term weather and climate forecasts. Medium- and longterm forecasts are needed to predict the efficiency of thermal and solar PV plants under various weather conditions and also help predict the contribution of generation from variable renewable energy sources (such as wind and solar PV) (Miara et al. 2017; FERC 2018). Rainfall and temperature forecasts also help inform hydropower reservoir operation and planning decisions. Grid operators can better understand how weather events and trends may impact the larger system and then identify the optimal generation mix to meet demand for specific years with these medium- and long-term forecasts.

Power system models may be coupled with transmission and distribution system models and knowledge of extreme weather events to evaluate impacts on transmission and distribution systems, such as the impact of extreme temperatures on the efficiency of these systems. Dehghanian et al. (2019) note that weather-driven generation and demand models can be combined to simulate electricity markets under various weather conditions. This allows system operators to more accurately project price and overall wholesale market impacts from expected weather events, potentially avoiding congestion or supply shortages.

Access to high-quality data can also support power sector resilience decision making and inform planning and training. Examples may include:

- Acquiring high-resolution data on climate trends and relevant natural, technical, and human-caused threats (see Box 2)

- Identifying power sector vulnerabilities, threats, and likely impacts if exposed

- Characterizing interdependencies of power sector-reliant systems that could be impacted (e.g., populations, communications, transportation, health systems, and government operations)

\section{Colombia Utility Enhances Resilience Through \\ Grid Awareness and Flexibility}

Operational intelligence is an important factor in supporting power system resilience, as it allows for increased grid awareness and flexibility during extreme events or outages. The use of widearea controls that enable increased communication and measurement across the power system is one example. With these controls, operators can intentionally island or separate parts of the grid in response to changing conditions or a potential threat.

By rapidly identifying urgent risks to systems, increased operational intelligence enhances resilience through decreased decision time. Ideally, this is accomplished by coupling artificial intelligence systems with human-interfacing systems to aid grid operators in focusing on the most urgent risks.
(National Academies of Sciences, Engineering, and Medicine 2017). XM, the grid operator of Colombia, has included this type of mixed system in its state-ofthe-art control center in the city of Medellin. A similar, potentially lower-cost solution is to increase staffing to ensure job-function redundancy across the power sector. This may include hiring duplicate staff members to perform specific job functions or cross-training staff to perform operations outside their typical role. In either case, this increases flexibility of staffing during events that strain resources. XM has also instituted this level of redundancy for specific job functions to ensure resilient grid operations throughout Colombia (XM Control Center Staff 2018).

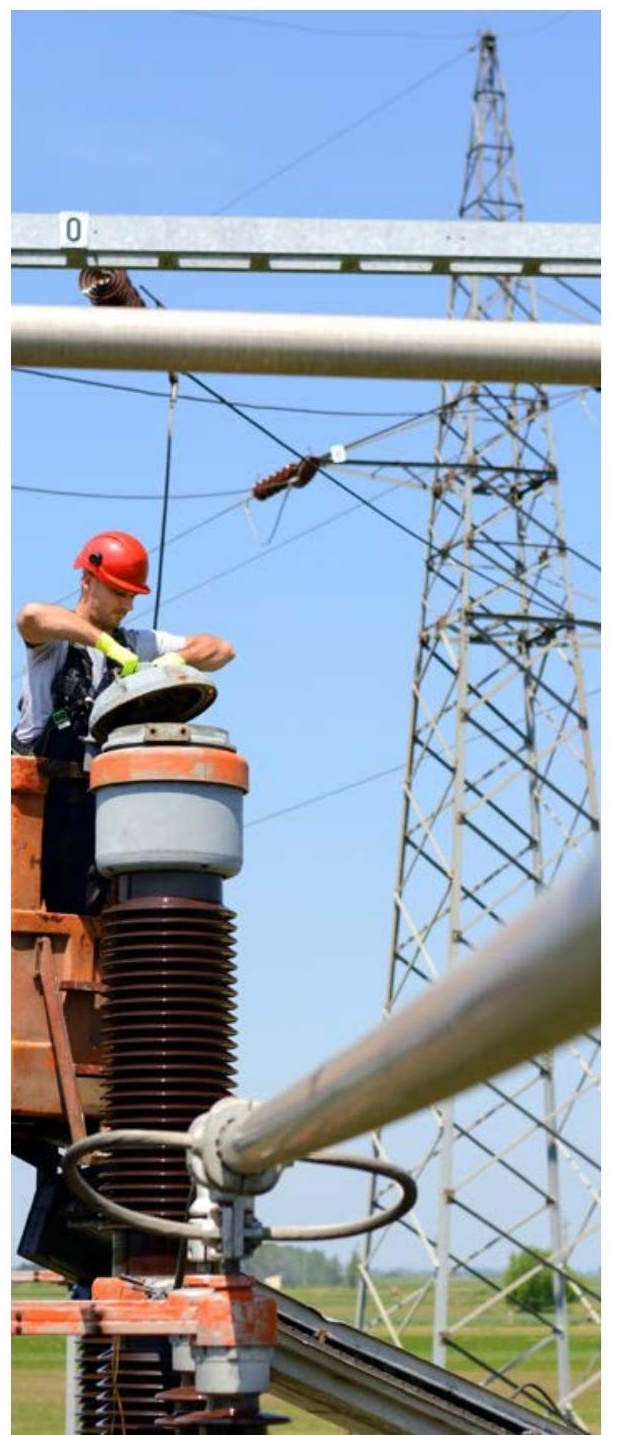


- Creating an inventory of potential mitigation actions and relevant technologies together with associated costs and benefitsincluding mitigation actions that have multisectoral impacts

\section{- Developing a consistent}

methodology to evaluate and prioritize mitigation actions that supports continued evaluation of resilience solutions in a changing environment (DOE 2013).

\section{Enhance Operational Intelligence}

Operational intelligence refers to understanding and preparing for how threats may impact the physical infrastructure of the system as well as the operational system (such as control centers and communication networks), personnel (such as management, technical staff, and their families), and finances (such as utilities' access to funds to continue operations).

Ensuring that critical repair personnel are strategically located and can communicate effectively to respond rapidly and access equipment that requires repair after an event is one example of operational intelligence. This is enhanced by also ensuring that vital spare parts (such as distribution poles, cables, and transformers, among others) are stored and staged in strategic locations to ensure they are accessible to personnel during responses (see Box 3). Additional measures may include diversifying the supply chains of parts, fuels, and equipment to mitigate risks during extreme events.

Power systems are rapidly introducing new "smart" systems that include customer meters, distributed energy resources, and automated grid controls. While these technologies can enhance resilience through grid flexibility and shortened response times, they can also increase vulnerability to cyberattacks. Operational changes that include increased use of smart technologies must also include adoption of cyberresilient practices such as situational awareness training for staff, consistent monitoring of cyberthreats, appropriate data management, and planned responses to attacks. Box 4 describes how strategies can also be implemented to increase operational intelligence of systems through wide-area monitoring and controls as well as redundant staffing and operations.

Along with contingency plans to keep operational systems online, flexible business continuity plans are also necessary to ensure that organizations

5. Norwegian Hydropower Company Uses

Collaborative Approaches to Support Resilience

Statkraft, a hydropower generation company in Norway, relies heavily on hydrological forecasting models to ensure it has reliable and timely data for decision making. To support these models, Statkraft has a network of more than 120 hydro-meteorological observation stations within its operational mountain basin areas. Statkraft has a long history of working with the Norwegian Meteorological
Institute to improve the shortterm forecasting that supports the company's hydropower operations. On the working level, this means an expert representative from the Metrological Institute is present every morning at Statkraft's forecasting center to assist with reviewing data and developing special forecasts to support Statkraft's hydropower generation planning activities (WBCSD 2014). can adapt to changing conditions and recover quickly during disruptions (National Academies of Sciences, Engineering, and Medicine 2017; Wang et al. 2016; Queensland Reconstruction Authority 2011).

Improving operational intelligence also requires periodic review and evaluation of existing plans and solutions to ensure that they are correctly implemented, that they are effective, and that they are applicable to current practices and changing environmental conditions. These evaluations provide an opportunity to identify shortcomings in solutions, resolve these shortcomings, and inform future resilience planning activities.

\section{Engage Stakeholders}

Intergovernmental communication, coordination, and preparation are critical first steps to resilience planning, and the multijurisdictional nature of power sector planning requires the convening of a broad stakeholder group to lead power sector resilience planning and contribute to a diverse set of perspectives (Hotchkiss et al. 2018). These relationships will support rapid responses and allow for streamlined processes in the event of an emergency, such as ensuring better communication between distribution systems operators and local governments in the event of a flood or hurricane (Queensland Reconstruction Authority 2011). Box 5 presents an example of collaborations that support power sector resilience.

Stakeholder engagement includes the development of relationships, partnerships, and cooperation at different institutional levels as well as with the broader public that improve communication channels for emergency response and holistic power sector resilience planning (Wang et al. 2016). Box 6 discusses how a broad set of stakeholders can be engaged to support power sector resilience planning. 
Engagement also includes public outreach efforts to build awareness about power sector resilience. Effective and safe public response to disasters and disruptions will enable power sector managers to communicate more effectively with their users and encourage appropriate end-user behavior during extreme events. This may include ensuring populations avoid downed power lines and disconnect systems that may be affected by water until they are inspected by qualified professionals. Public awareness also includes developing an understanding of why the power outages occur, and how this may include preemptive disconnections by utilities to protect systems and allow for rapid restoration of services after a threat has passed.

\section{Develop a Culture of Resilience}

Leadership-level support and inspiration for taking on challenges, adapting, innovating, and seeking new opportunities are vital to developing a culture of resilience. This may include supporting continued research, development, and demonstration of technical and institutional solutions as well as identifying and removing technical and bureaucratic barriers that may impede transitions to more resilient energy sectors (Foster 2017; DOE 2013). Leadership can also effect changes and organizational policies that support organizational resilience (Box 7).

Ensuring that personnel understand both the motivation to enhance power sector resilience and their role in supporting these activities will help to include knowledgeable staff in the implementation of solutions as well as the identification of potential threats, vulnerabilities, and resilience solutions. This also supports knowledge and capacity-building of personnel.

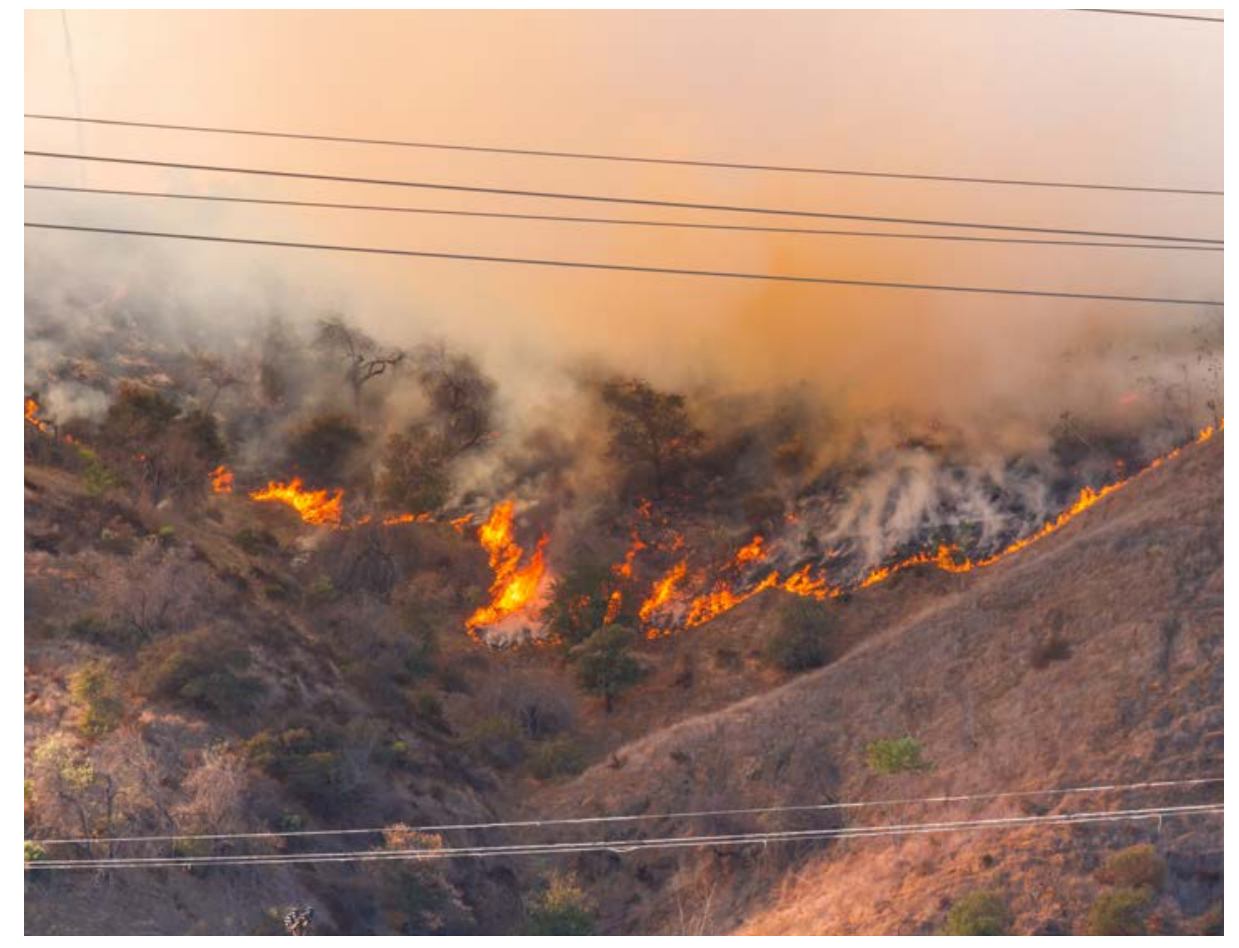

\section{Lao PDR Leverages Stakeholder Engagement and Relationships for Power Sector Resilience Planning}

The Ministry of Energy and Mines of the Lao People's Democratic Republic (Lao PDR) recognizes that access to reliable, secure, and affordable electricity is essential to achieving the country's objectives. The power system plays a vital role in the provision of power, but it also faces potential risks from natural, technological, and humancaused threats. To address this and enhance power sector resilience, the Ministry of Energy and Mines (MEM) undertook a comprehensive national power sector vulnerability assessment and resilience action planning activity in 2018. The Ministry engaged first-of-theirkind advisory and stakeholder groups to support this work.

The advisory group, which included power sector directors/ managers from MEM, Électricité du Laos (EDL), and EDL-Generation Public Company (EDL-Gen), provided strategic direction. The wide-ranging stakeholder group included representatives from the Ministry of Industry and Commerce, Ministry of Science and Technology, Ministry of Natural Resources and Environment, Ministry of Finance, Ministry of Labor and Social Welfare, Lao Holding State Enterprise, Central Bank of Lao PDR, and the Lao Women's Union.

Broad stakeholder engagement helped to capture diverse experiences and perspectives from across the power system. Stakeholder engagement also built local technical capacity for resilience planning, engaged multiple stakeholders from across the government to help build consensus, and ensured local expertise and institution knowledge would be available to support future resilience planning. 


\section{Resilient Energy Platform Targets Power Sector Vulnerabilities}

The Resilient Energy Platform helps countries to address power system vulnerabilities by providing strategic resources and direct country support to enable planning and deployment of resilient energy solutions. The platform

\section{New Zealand Firm Takes Steps to Ensure Well-being of Personnel to Enhance Emergency Response}

Following a 2011 earthquake in Christchurch, New Zealand, which impacted the safety of local network maintenance service provider Connetics' personnel, leadership developed personalized profiles of all their personnel and families. These profiles would allow Connetics to support the professional and personal needs of its personnel (such as providing shelter, food, water, and emotional support) during extreme eventsenabling them to work during extended shifts while allowing Connectics to support their needs during emergencies (Wang et al. 2016). includes expertly curated reference material, training materials, data, tools, and direct technical assistance in planning resilient, sustainable, and secure power systems. Ultimately, these resources enable decision makers to assess power sector vulnerabilities, identify resilience solutions, and make informed decisions to enhance energy sector resilience at all scales (including local, regional, and national scales).

To learn more about the organizational solutions highlighted in this fact sheet, please visit the platform at www. resilient-energy.org.

\section{References and Resources to Learn More}

Dehghanian, Payman, Bei Zhang, Tatjana Dokic, and Mladen Kezunovic. 2019. "Predictive Risk Analytics for Weather-Resilient Operation of Electric Power Systems." IEEE Transactions on Sustainable Energy 10 (1): 3-15. https://doi.org/10.1109/TSTE.2018.2825780.

DOE. 2013. U.S. Energy Sector Vulnerabilities to Climate Change and Extreme Weather. Washington, D.C.: U.S Department of Energy (DOE). DOE/PI-0013. https://www energy.gov/downloads/us-energy-sector-vulnerabilities-climate-change-and-extreme-weather.

FERC. 2018. “Comments of the California Independent System Operator Corporation in Response To The Commission's Request for Comments About System Resiliency and the Threats To Resilience." Grid Resilience in Regional Transmission Organizations and Independent System Operators, Federal Energy Regulatory Commission (FERC) Docket No. AD18-7-000. http://www.caiso.com/

Documents/Mar9_2018_Comments-GridResilience_ AD18-7.pdf.

Foster, Vivian. 2017. Meeting the Challenges of Enhancing Power-Sector Resilience. Presentation delivered at the Asian Clean Energy Forum, Manila. https:/ d2oc0ihd6a5bt.cloudfront.net/wp-content/uploads/ sites/837/2017/06/5_Meeting-the-Challenges-ofEnhancing-Power\%E2\%80\%93-Sector-Resilience-.pdf.
FPL. 2019. "Our Comprehensive Storm Plan Focuses on Readiness, Restoration, and Recovery." Florida Power and Light Company (FPL) Storm Center: How We Prepare. https://www.fpl.com/storm/how-we-prepare.html.

Hotchkiss, Eliza, Alex Dane, Connie Komomua, and NREL. 2018. "Resilience Roadmap: A Collaborative Approach to Multi-Jurisdictional Planning." National Renewable Energy Laboratory (NREL). https://www.nrel.gov/

resilience-planning-roadmap

Insurance Journal. 2011. Storm Joachim Hits Northern Europe; AIR Sees No 'Significant' Losses. Insurance Journal. December 20. https://www.insurancejournal.com/news/ international/2011/12/20/228102.htm.

Miara, Ariel, Jordan E. Macknick, Charles J.Vörösmarty, Vincent C. Tidwell, Robin Newmark, and Balazs Fekete. 2017. Climate and water resource change impacts and adaptation potential for U.S. power supply. Nature Climate Change 7 (Oct.): 793.

National Academies of Sciences, Engineering, and Medicine. 2017. Enhancing the Resilience of the Nation's Electricity System. Washington, DC: The National Academies Press. https://doi.org/10.17226/24836.

Queensland Reconstruction Authority. 2011. Planning for stronger, more resilient electrical infrastructure: Improving the resilience of electrical infrastructure during flooding and cyclones. Brisbane: Queensland Reconstruction Authority. https://www.statedevelopment.qld.gov.au/ resources/guideline/qra/planning-resilient-electrical-infrastructure.pdf

Wang, Xiaoping, Ray Brown, Guillaume Prudent-Richard, and Katrina O'Mara. 2016. Enhancing Power Sector Resilience Emerging Practices to Manage Weather and Geological Risks. Washington, DC: World Bank. https:// openknowledge.worldbank.org/handle/10986/26382.

WBCSD. 2014. Building a Resilient Power Sector. Geneva: World Business Council for Sustainable Development (WBCSD) Electric Utilities. https://www.wbcsd.org/ Programs/Climate-and-Energy/Climate/Resources/ Building-a-Resilient-Power-Sector.

World Bank. 2016. Are Power Utilities in Tonga and New Zealand Resilient? Human and Organizational Factors in Disaster Response. LiveWire: A Knowledge Note Series for the Energy \& Extractives Global Practice. https://openknowledge.worldbank.org/bitstream/handle/10986/23634/ Are0powerOutil0inodisasterOresponse.

pdf? sequence $=1$ \&is Allowed $=\mathrm{y}$.

XM Control Center Staff. 2018. Author discussions and visit to XM Control Center of the National Dispatch System, Medellin, Colombia.

Photos from iStock. Page 2: 881926684; page 3: 930547052; page 4: 475211706; page 7: 1065674238

Written by Nathan Lee, Sherry Stout, and Sadie Cox, National Renewable Energy Laboratory (NREL), with editorial support and consulting from Kate Anderson, Alicen Kandt, Andrea Watson, Adam Warren (NREL) and Jennifer Leisch (USAID).

www.resilient-energy.org | www.nrel.gov/usaid-partnership

Jennifer E. Leisch, Ph.D.

USAID-NREL Partnership Manager

U.S. Agency for International Development Tel: +1-303-913-0103 | Email: jleisch@usaid.gov

\section{Sadie Cox}

Senior Researcher

National Renewable Energy Laboratory

Tel: +1-303-384-7391 | Email: sadie.cox@nrel.gov

This work was authored, in part, by the National Renewable Energy Laboratory (NREL), operated by Alliance for Sustainable Energy, LLC, for the U.S. Department of Energy (DOE) under Contract No. DE-AC36-08GO28308. Funding provided by the United States Agency for International Development (USAID) under Contract No. IAG-17-2050. The views expressed in this
report do not necessarily represent the views of the DOE or the report do not necessarily represent the views of the DOE or the
U.S. Government, or any agency thereof, including USAID.

NREL/TP-7A40-73645 | May 2019

NREL prints on paper that contains recycled content.
The Resilient Energy Platform provides expertly curated resources, training, tools, and technical assistance to enhance power sector resilience. The Resilient Energy Platform is supported by the U.S. Agency for International Development.

The USAID-NREL Partnership addresses critical challenges to scaling up advanced energy systems through global tools and technical assistance, including the Renewable Energy Data Explorer, Greening the Grid, the International Jobs and Economic Development Impacts tool, and the Resilient Energy Platform. More information can be found at: www.nrel.gov/usaid-partnership.

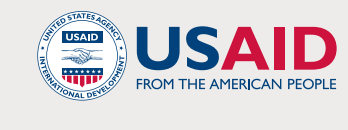
MNREL

REexplorer
MAPPIng OUR ENERG Future

